\title{
Direct and Broadband Plasmonic Charge Transfer to Enhance Water Oxidation on a Gold Electrode
}

\author{
Matthias Graf,* Gregor B. Vonbun-Feldbauer, and Marc T. M. Koper \\ Cite This: ACS Nano 2021, 15, 3188-3200 \\ Read Online
}

\section{ACCESS \\ Llll Metrics \& More \\ Article Recommendations \\ Supporting Information}

ABSTRACT: Plasmonic photocatalysis via hot charge carriers suffers from their short lifetime compared with the sluggish kinetics of most reactions. To increase lifetime, adsorbates on the surface of a plasmonic metal may create preferential states for electrons to be excited from. We demonstrate this effect with $\mathrm{O}$ adsorbates on a nanoporous gold electrode. Nanoporous gold is used to obtain a broadband optical response, to increase the obtained photocurrent, and to provide a SERS-active substrate. Only with adsorbates present, we observe significant photocurrents. Illumination also increases the adsorbate coverage above its dark potential-dependent equilibrium, as derived from a two-laser in situ SERS approach. Density functional theory calculations confirm the appearance of

Light shifts adsorption equilibrium on $\mathrm{Au}$

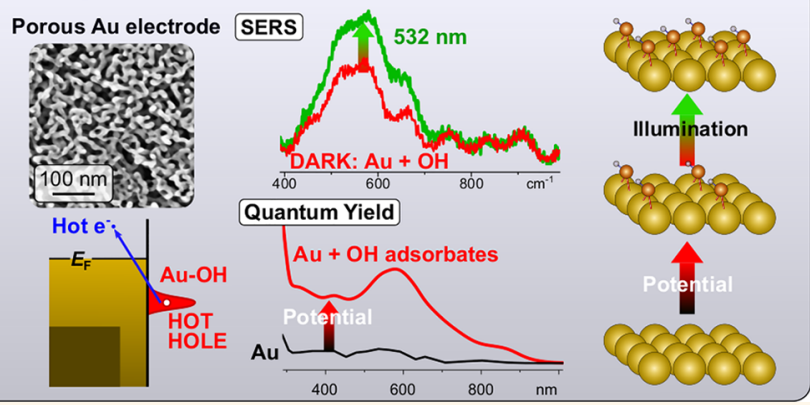
excitable states below the Fermi level. The photocurrent enhancement and broadband characteristics reveal the potential of the plasmonic approach to improve the efficiency of photoelectrochemical water splitting.

KEYWORDS: chemical interface damping, density functional theory, hot electron, hot hole, nanoporous gold, photoelectrocatalysis, surface-enhanced Raman spectroscopy

$\mathrm{P}$ hotoelectrochemistry is currently considered the most promising strategy towards a green and efficient solar $\mathrm{H}_{2}$ fabrication via water splitting. ${ }^{1,2}$ Alternatively to established semiconductor-based approaches, harvesting of solar power and with this locally separating charges via a plasmonic, that is, metallic photon absorber approach, is currently under intense debate. Among several specific challenges, one general bottleneck of the water splitting reaction is the sluggish anodic oxidation reaction of oxygen from water. ${ }^{3-6}$

In plasmonic water splitting, excited charge carriers shall couple into the individual partial reactions. In detail, the absorption of a photon creates an excited, or hot, electron which can reduce a proton or water molecule. In an anodic reaction, the remnant of electron excitation, called hot holes, oxidizes hydroxide ions or water. Both processes are determined by the damping of the metal's plasmon polariton and hence the absorption mechanism. ${ }^{7,8}$ Upon photon absorption in metal nanostructures, the energy of one photon can lead to the excitation of either a free sp-band or a localized d-band electron over the metal's Fermi energy to form a hot electron. ${ }^{9}$ When neglecting field enhancement effects, this excitation to an electron distribution far from equilibrium can occur via different mechanisms: by electron collisions with a phonon or impurity, by electron-electron scattering, by interband transmission at high photon energies, or by collisions with the surface, often referred to as Landau damping. ${ }^{7,10,11}$ Hot electrons have a short lifetime, which is determined by the scattering modes with other "cold" electrons. ${ }^{12}$ Subsequent relaxation by further scattering leads to a bath of thermalized or "lukewarm" carriers which will further relax to finally heat the metal lattice. ${ }^{11}$ It is currently under intense debate whether already found photoenhancements of certain reactions are due to carrier transfer effects or to enhanced reaction kinetics as a consequence of an elevated surface temperature. ${ }^{10,13,14}$

To utilize hot carrier species for heterogeneous chemical reactions several other points must be regarded: (1) the energy

Received: November 21, 2020

Accepted: January 21, 2021

Published: January 26, 2021 
level $\varepsilon$ of the species accepting the carrier must be compatible with the energy level of the excited electron or hole after absorption, that is, for electrons, this must be $\varepsilon \leq \varepsilon_{i}+\hbar \nu$ (with the initial energy level of the electron before excitation, $\varepsilon_{i}$, Planck's constant, $\hbar$, and the frequency of the photon, $\nu$ ) and for holes this will be $\varepsilon \geq \varepsilon_{i}{ }^{15,16}$ When hot electrons are injected into water to drive the reduction of free protons in solution, $\varepsilon$ is the energy of water's conduction band $\left(\mathrm{CB}_{\mathrm{H}_{2} \mathrm{O}}\right)$ that electrons are emitted into. ${ }^{16-18}$ When an adsorbate is reduced, such as a thiol species, $\varepsilon$ is the energy of the lowest unoccupied molecular orbital (LUMO) formed as a consequence of hybridization between the electronic states of the metal and the molecule. ${ }^{15,19}$ This concept similarly applies to oxidation reactions with holes being emitted into the valence band of the surrounding medium, for example, water, or if an adsorbate, for example, citrate, is present to be oxidized, the relevant energy level is its highest occupied molecular orbital (HOMO). ${ }^{20}$ (2) The average reaction time needs to be shorter than the lifetime of both carriers before thermalization excludes thermal effects as the origin for enhancement. While the latter is in the range of $10-100 \mathrm{fs}$, the kinetics of chemical reactions are usually much slower. ${ }^{12,19,21}$ (Note that electrons predominantly relax via electron-electron collisions, and their mean free path is $10-100 \mathrm{~nm}$. With a Fermi velocity of $1.4 \times 10^{8} \mathrm{~cm} \mathrm{~s}^{-1}$, this yields a mean lifetime of $10-100 \mathrm{fs}$.) Electrochemistry enables to significantly increase the reaction rate and hence decrease the reaction time by applying a certain potential to the metal. An overpotential of $1 \mathrm{~V}$ can be estimated to lead to a drop in this time to $17 \mathrm{fs}$ for one proton to be converted on a Au electrode, that is, within the lifetime of a hot electron, whereas the same reaction takes approximately $5 \mu \mathrm{s}$ at zero overpotential. ${ }^{23}$ (Rate constants are estimated via Butler-Volmer equation with a charge-transfer coefficient of 0.5 , room temperature, and an exchange current density of $10^{-6} \mathrm{~A} \mathrm{~cm}^{-2}$. The reaction times were determined based on a bulk proton concentration of $1 \mu \mathrm{M}$ which is decreased by up to $4.3 \mathrm{pH}$ when approaching the electrode-electrolyte interface $^{\frac{22}{2}}$ ). Simultaneously, this overpotential causes electrolytic reactions to occur in parallel, that is via the transfer of nonexcited carriers, so that strong photoelectrochemical reaction enhancements are frequently accompanied by dark currents. $^{10,24}$

The available values for chemical reaction plasmonic enhancements are still consistently low due to manifold reasons: a high transfer barrier defines a wavelength selectivity, high recombination rates of hot carriers limit the number of transferred carriers, excitation of d-band electrons leads to absorbed photons being "wasted" for generating unreactive carriers, etc. ${ }^{16,25-27}$ Increasing the lifetime of hot carriers could drastically improve the chance for them to contribute to the conversion. Meanwhile, higher currents would be connected to a decrease in overpotential (for the same reaction rates) of a certain reaction. Lifetime prolongation can be realized by injecting hot carriers into an adjacent phase, for example, a semiconductor, but this approach requires another transfer process between the semiconductor and the electrolyte where the chemical reaction takes place. ${ }^{28}$ Although high injection efficiencies of up to $45 \%$ were found for such injection from $\mathrm{Au}$ into $\mathrm{TiO}_{2}$, the final transfer from the semiconductor into water typically occurs (under dark conditions) at significantly smaller rates than at a metal-electrolyte interface so that the total efficiency is expected to be still very low. ${ }^{12,28-30}$
Alternatively, other excitation-transfer schemes were proposed which are promising to prolong the lifetime of hot carriers and couple them directly with chemical reactions. Among them, a modified absorption-excitation process was identified when adsorbate molecules are attached to the metal surface (e.g., by electrochemical adsorption or a preceding chemical modification processes), so-called chemical interface damping (CID). CID is a plasmon damping channel which becomes more important with increasing surface-to-volume ratio (SVR). ${ }^{31-33}$ In contrast to Landau damping, CID might lead to a charge dislocation between the metal and the adsorbate with the carrier being located in both phases with similar probability. ${ }^{34}$ This process is often considered as a direct charge transfer and occurs from the metal to an unoccupied hybrid molecular state of the adsorbate to produce a hole in the metal. ${ }^{35-37}$ The opposite process, the excitation of an electron from the hybrid-HOMO of the adsorbate to be directly transferred into the metal creating a reactive hole in the hybrid state, was proposed theoretically, but experimental evidence for this transfer process is still lacking. ${ }^{33,38}$ The hole in the hybrid state could participate very efficiently in the reaction since its lifetime is significantly larger.

Creating hot carriers directly at the interface will lead to highly reactive species. This process benefits from high-SVR structures. ${ }^{16,17,31,39}$ In the past, more or less significant reaction enhancements by hot carriers were frequently demonstrated by a nanoparticle approach within which spectra of quantum efficiencies frequently showed maxima that coincided with the plasmon resonance effect. ${ }^{27,40-43}$ In addition, the variation of the nanoparticle size does not allow to accurately conclude on the influence of SVR variation since photoelectrodes cannot stably be covered with a defined number of nanoparticles. ${ }^{16} \mathrm{~A}$ surface effect, which can be identified from a plot of the internal quantum efficiency vs. SVR, and its benefit toward charge transfer could be accurately demonstrated if accurate SVR variation is given. ${ }^{16}$ In contrast, using a plasmonic, yet broadband absorbing material which is adjustable in its SVR would allow valid conclusions. While additionally increasing the total absorption, the plasmonic metamaterial of nanoporous $\mathrm{Au}(\mathrm{npAu})$ enables such SVR variation. It is constructed of a bicontinuous network of 15-40 vol.- $\%$ of nanoscale Au struts (called ligaments). ${ }^{44}$ These are separated by pores that, when used as an electrode, contain the liquid electrolyte so that $\mathrm{npAu}$ provides a large contact area through the high SVR ${ }^{45}$ Furthermore, npAu generally exhibits a reactivity which is higher than one would expect for flat polycrystalline Au surfaces (hence, reaction times are lower than at other $\mathrm{Au}$ surfaces so that more hot carriers could couple into chemistry), while it shows its chemical stability, besides a structural stability over a large range of conditions. $^{46-49}$ When npAu was applied as an emitter material of hot electrons, its accurate SVR adjustability previously enabled the separation of bulk and surface photon absorption and electron emission. ${ }^{16}$ Being used as a photoelectrode material within a photoelectrochemical approach, its high metallic conductivity and its ligand-free surface $^{50-52}$ enable addressing specific reactions via the applied potential as well as an accurate quantitative determination of exclusively charge-transfer events via the electrochemical current. ${ }^{53}$

Herein, we present experimental and theoretical evidence for a direct charge transfer from an adsorbate state into the metal on the broadband-absorbing plasmonic photoelectrode made from npAu. We correlate values of photocurrent as a function 
of its feature size, potential, wavelength, and power combined with surface characterization via in situ two-laser surfaceenhanced Raman spectroscopy (in situ 2L-SERS). This approach screens the changes that occur at the surface during the photoelectrochemical process and that are responsible for the photocurrent. As both photocurrent and in situ 2L-SERS intensity show an excellent congruence in their power, potential, and wavelength dependences, we derive a mechanistic picture for an adsorbate-mediated direct charge-transfer process. In contrast to previous approaches, this effect is efficient over the entire visible wavelength range.

\section{RESULTS AND DISCUSSION}

Samples. $100 \mathrm{~nm}$-thin layers of $\mathrm{npAu}$ were produced from white gold $\left(\mathrm{Ag}_{75} \mathrm{Au}_{25}\right)$ leaves via potentiostatic dealloying as reported earlier. ${ }^{54}$ The obtained porous sheets were stripped onto a carrier material to use them as electrodes in a photoelectrochemical cell (see Methods section for details). SEM images of the electrodes (see Figure SI-1 left column) show the typical bicontinuous porous mesostructure of ligaments and pores. As can be seen from Figures $1 \mathrm{~A}-\mathrm{D}$ and SI- 1 , their sizes could be increased in a stepwise manner via thermal coarsening at $300{ }^{\circ} \mathrm{C}$. The electrodes were not
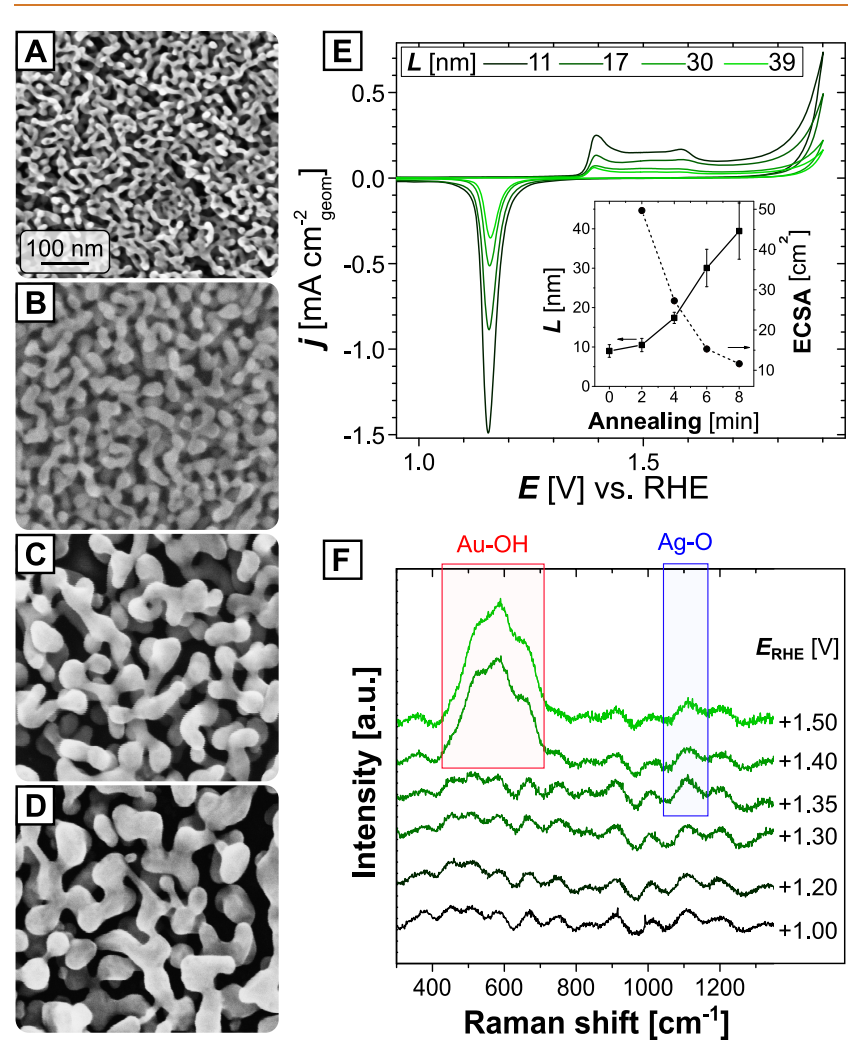

Figure 1. Properties of the investigated npAu electrodes. (A-D) Scanning electron micrographs of samples annealed at $300{ }^{\circ} \mathrm{C}$ for 2 (A), 4 (B), 6 (C), and $8 \mathrm{~min}$ (D) from which average ligament diameters $L$ were determined (see Figure SI-1 for details). (E) Cyclic voltammograms of npAu electrodes (scan rate: $10 \mathrm{mV} \mathrm{s}^{-1}$ ) in $0.5 \mathrm{M} \mathrm{H}_{2} \mathrm{SO}_{4}$ electrolyte. Inset: $L$ (left axis) and the electrochemically active surface area (ECSA, right axis) as determined from the hydroxide adsorption peak in the positive scan (see Methods section for details) vs. annealing duration. (F) In situ SER spectra of npAu (after 2 min annealing) with applied potential. Relevant peaks were attributed based on literature and are marked according to their first evolution. ${ }^{55,56}$ disintegrated by this process, that is, we observe no ligament rupture, no cracking of the porous thin layer, and no film delamination (see Figure SI-1) so that the volumetric shrinkage through coarsening is negligible and the electrodes can be considered as stable simply being varied in their SVR.

The electrodes were further characterized by cyclic voltammetry $(\mathrm{CV})$. Figure $1 \mathrm{E}$ shows the typical features of $\mathrm{Au}$ (hydr)oxide formation (in the positive scan) up to the evolution of molecular $\mathrm{O}_{2}$ (oxygen evolution reaction, OER) and, upon reversing the scan direction, $\mathrm{Au}$ (hydr)oxide reduction. The height of all these signals depends on the average ligament diameter $L$ (for determination see Methods section), that is, larger ligaments lead to lower currents since the active surface area is decreased by coarsening. The inset in Figure $1 \mathrm{E}$ shows the average ligament size $L$ in its evolution during the coarsening process. The integration of the oxidation current in the $\mathrm{CV}$ delivers a charge. Based on a surface oxidation mechanism under acidic conditions as suggested by Conway et al., one can define a potential $E_{\theta=1}$ at which a full monolayer of $\mathrm{OH}$ is formed. ${ }^{57}$ As explained in detail in Section SI-B, we further assume that in a potential range $1.35 \mathrm{~V} \leq E \leq$ $1.44 \mathrm{~V}$ (all potentials in this manuscript refer to the reversible hydrogen electrode, RHE), the dominant adsorbate species on $n p A u$ is $\mathrm{OH}$ until a complete $\mathrm{OH}$ coverage is achieved at $E_{\theta=1}$ $=1.44 \mathrm{~V}$. In $1.44 \mathrm{~V}<E \leq 1.63 \mathrm{~V}$, it is $\mathrm{O}$, in $1.63 \mathrm{~V}<E \leq 1.80$ $\mathrm{V}$, a multilayer adsorption takes place, and when $E>1.80 \mathrm{~V}$, OER sets on. Please compare with the literature for a more detailed description. ${ }^{55}$ The charge corresponding to a monolayer gives an estimate of the ECSA (see Section SI-B and Figure SI-2 for details). As expected, the ECSA is found to decrease when the structures are coarsened according to ECSA $\sim L^{-1}$ (right axis in the inset of Figure 1E).

In situ SERS allows characterization of surface species that evolve during the application of an electrochemical potential (see Methods section and Section SI-C for details). ${ }^{55,58}$ SER spectra of as-dealloyed npAu stripped onto a Au carrier (Figure SI-3) show peaks at 360 and $1110 \mathrm{~cm}^{-1}$, which become stronger than the background noise above $1.35 \mathrm{~V}$. These peaks can be attributed to different vibrational modes of $\mathrm{Ag}_{2} \mathrm{O}$, a remnant from the dealloying process. ${ }^{56}$ After a short annealing step (within which $L$ and, hence, the SERS enhancement factor do not change significantly), both Ag peaks cannot be identified clearly anymore (Figure $1 \mathrm{~F}) .{ }^{59}$ In both samples, one finds a broad peak between 405 and $700 \mathrm{~cm}^{-1}$ which sets in between 1.35 and $1.4 \mathrm{~V}$. This peak results from an overlap of several geometrically slightly different vibrational modes of the $\mathrm{Au}-\mathrm{O}$ bond along a strongly curved surface. ${ }^{60}$ Together with the above-mentioned mechanism of Au oxidation, we attribute it to $\mathrm{Au}-\mathrm{OH}$ species at the surface. Note that a separation between $\mathrm{Au}-\mathrm{OH}$ and $\mathrm{Au}-\mathrm{O}$ cannot be expected under the present conditions. ${ }^{60}$ The potential at which the $\mathrm{Au}-\mathrm{O}$ signal evolves coincides with the onset potential $E_{\text {ads }}$ of surface oxidation observed during CV (Figure SI-2D) and is consistent with that found for flat Au electrodes. ${ }^{55}$

It is known that dealloying of a $\mathrm{Ag}-\mathrm{Au}$ alloy is a process in which $\mathrm{Ag}$ dissolution and Au surface diffusion are balanced. ${ }^{61}$ Consequently, if $\mathrm{Ag}$ is not removed completely, it remains in npAu within Ag-rich clusters which are "buried" by at least one monolayer of Au atoms. ${ }^{48}$ Sub-surface Ag is known to increase the oxophilicity ${ }^{62}$ of $\mathrm{npAu}$, which is a possible origin for its high catalytic activity. ${ }^{63,64}$ However, under annealing conditions, Ag-rich clusters were also found to dissolve so that the $\mathrm{Ag}$ concentration homogenizes over the entire structure and 

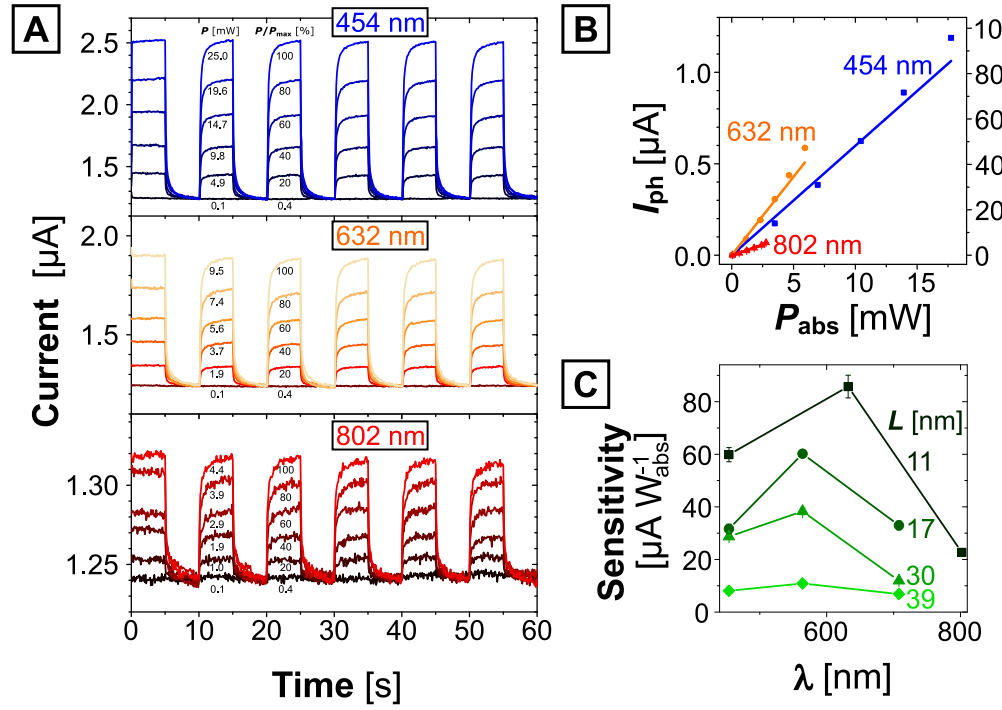

Figure 2. Results of the photocurrent measurements on annealed $(2 \mathrm{~min}) \mathrm{npAu}(\mathrm{A}$ and B with ligament size $L=11 \mathrm{~nm})$. (A) Current transients at a static potential of $E=1.4 \mathrm{~V}$ (vs. RHE) at chopped illumination (positive peaks correspond to the current under illumination) at different illuminating wavelengths for different incident powers $\boldsymbol{P}$ according to the available power $\boldsymbol{P}_{\max }$ of the instrument (see Figure SI-5 for its emission spectrum). (B) Obtained photocurrents $I_{\mathrm{ph}}$ (left axis, for details of determination see section SI-E) vs. the absorbed power $P_{\text {abs }}(=P \times A$, with the absorption $A$ taken from the absorption spectrum in Figure SI-4) and the relative enhancement of the photocurrent compared to the base or dark current (right axis). (C) Slope of the linear regressions in (B) vs. wavelength $\lambda$ (denoted as sensitivity) for all different $L$, as indicated by the labels.
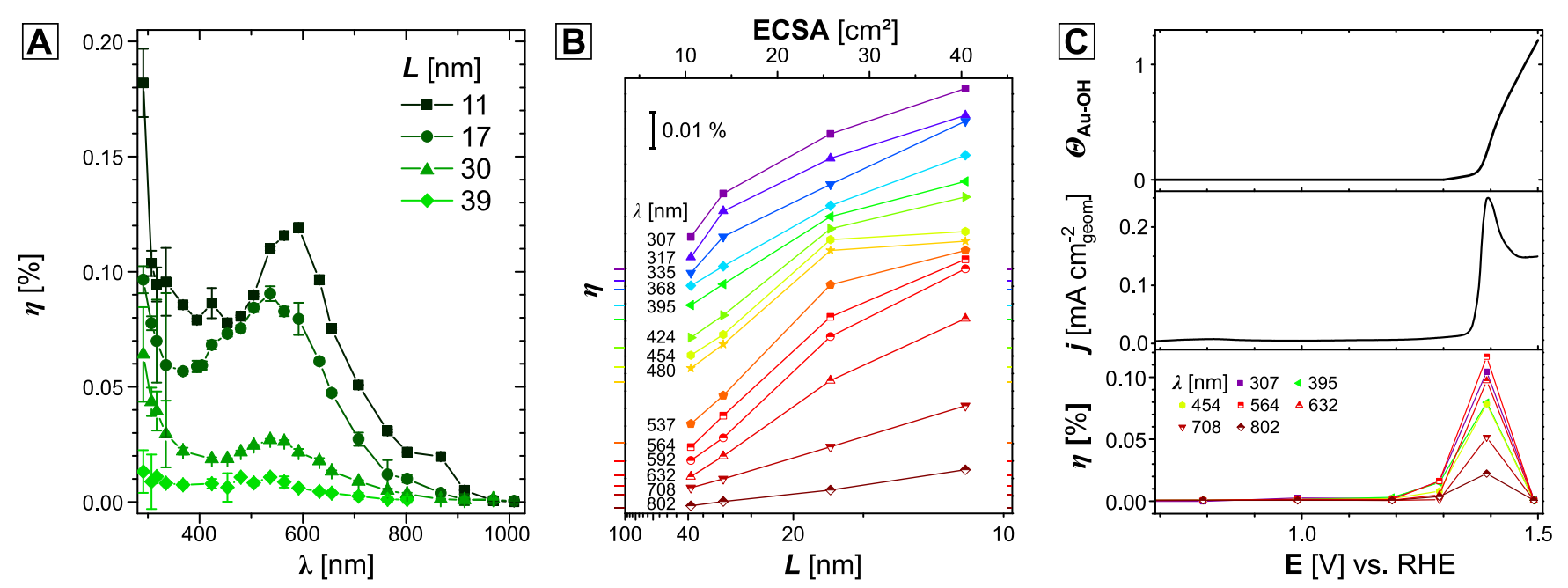

Figure 3. Dependence of photoanodic effect as expressed by the internal quantum efficiency $\boldsymbol{\eta}$ on (A) the wavelength $\lambda$ for different ligament diameters $L$ (all at $E=1.4 \mathrm{~V}$ vs. RHE). (B) The dependence of $\eta$ on the inverse of $L$ (which is proportional to the ECSA, top axis) for different $\lambda$. Data are displayed as offset stack to improve visibility. Unit scale is given and colored ticks on ordinate correspond to $\eta=0$. (C) On the applied potential $E$ (lower plot). Additional plots in (C) indicate the correlation with the signal from CV (middle plot, see Figure 1E) and the surface coverage, $\theta_{\mathrm{Au}-\mathrm{OH}}$ of npAu with $\mathrm{OH}$ as calculated therefrom (upper plot, for details see Section SI-B in Supporting Information, all data for npAu with $L=11 \mathrm{~nm}$ ).

the concentration gradient between ligament surface and volume becomes negligible at residual $\mathrm{Ag}$ concentrations $>8$ atomic \%. ${ }^{65}$ According to energy-dispersive X-ray spectroscopy (EDX), our samples contain 11-14 atomic \% of Ag (see Figure SI-1), that is, also the surface can be expected to expose this (or even a lower) concentration of Ag. In this light, we emphasize that the $\mathrm{Ag}_{2} \mathrm{O}$ species are found only in the sample state after dealloying, that is, without an annealing step. On the other hand, we can conclude that annealed samples expose a negligible amount of Ag to the metal-electrolyte interface, that is, in the top-most or a sub-surface layer. For this reason, the further examination of the photochemical processes of adsorbate formation and hole transfer shall therefore be limited to these "clean" Au surfaces.

Photocurrent. As previously identified and as it can be observed in the absorption spectra in Figure SI-4 (see Section SI-D), the feature size $L$ represents a parameter to tune the broadband absorption property of npAu in its absolute height, yet not in its spectral features, that is, spectral shifts as observed earlier for rather incomparable npAu systems ${ }^{16,66-68}$ cannot be concluded. Electrodes with varying $L$ were employed as photoanodes in a three-electrode photoelectrochemical cell. Anodic photocurrents were measured in the same way as in the previous report, that is, at constant 

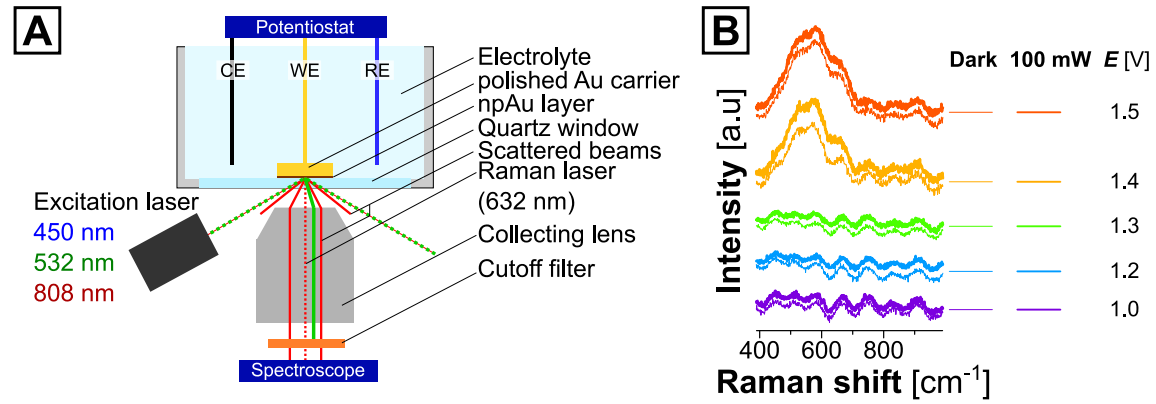

Figure 4. 2L-SER spectroscopy. (A) Schematic principle of experimental setup. (B) Comparison of SER spectra on a npAu electrode with $L=$ $11 \mathrm{~nm}$ at the respective (increasing) potential $E$ recorded without (thin lines) and under illumination (thick lines) with a $532 \mathrm{~nm}$ laser at $P=$ $100 \mathrm{~mW}$ as a proof-of-concept that illumination leads to higher coverages.

potential after a stable dark current had been established. ${ }^{16}$ As can be seen in Figure 2A, illumination within a broad wavelength $(\lambda)$ range leads to immediate and significant positive current jumps which are tunable by the respective light power. Values and errors of the photocurrent $I_{\mathrm{ph}}$ were determined based on the differences in the dark and light currents (see Methods section and Section SI-E for details) after averaging over six jumps (giving a standard deviation which we consider as the error in $\left.I_{\mathrm{ph}}\right)$. These are displayed as a function on the absorbed light power $P_{\text {abs }}(=P \times A(\lambda, L)$ with the incident source power $P$ and the $\lambda$ - and $L$-specific absorption $A$ as taken from Figure SI-4) in Figure 2B, including linear fits. It can be clearly seen that the slope of $I_{\mathrm{ph}}$ vs. $P_{\text {abs }}$ curve, which we denote as sensitivity, depends in a nonmonotonous way on $\lambda$. The dependence of the sensitivity on $\lambda$ is shown for all four samples in Figure 2C. Independent of $L$, Figure 2C (and the different wavelength ranges recorded for different values off $L$ ) suggests a maximum in sensitivity as a function of $\lambda$, with higher values of $L$ leading to smaller photocurrents and hence smaller sensitivity, as expected.

Sensitivity is a rather empirical measure which requires multiple measurements of $I_{\mathrm{ph}}$ at varied $P$ and is not capable of expressing the efficiency of a potential charge-transfer process. Instead, the number of absorbed photons $N_{\mathrm{abs}}$ that actually lead to an excitation of a number $N_{\mathrm{h}}$ of hot holes related to those which finally react gives the internal quantum efficiency $\eta$

$$
\eta=\frac{N_{\mathrm{h}}}{N_{\mathrm{abs}}}=\frac{I_{\mathrm{ph}}}{e} \times \frac{\hbar c}{\lambda A P}
$$

where $c$ is the speed of light in vacuum and $e$ is the elementary charge. Figure 3A illustrates spectra of $\eta$ for all samples. It is clearly visible that samples with smaller ligament structures lead to higher efficiencies over the entire $\lambda$ range.

A peak is observed between 537 and $592 \mathrm{~nm}$, which is still clearly visible for larger $L$. Although being located in the typical $\lambda$ range of the plasmon resonance of $\mathrm{Au}$, we do not attribute this peak to this effect. An attribution of absorption spectra (which do not exhibit a clear resonance feature in absorption, see Figure SI-4) to those of $\eta$ appears vague, but we find that the peak does not shift in position as one would expect from the size dependence of plasmon resonance effects. ${ }^{69}$ To still explain the origin of the peak, we suggest the following explanation: When decreasing $\lambda$, the absorption of d-band electrons contributes more and more to the total absorption and creates holes in the d-band. As will be discussed below, these do not contribute to the photocurrent so that a lower value of $I_{\mathrm{ph}}$ (see Figure 2) is normalized to still a high value of
$A$ (eq 1) and a drop in $\eta$ must result and to conclude the found peak. Upon further decreasing the wavelength below $395 \mathrm{~nm}$, values of $\eta$ again increase due to the increasing tendency to ballistically emit hot holes into the electrolyte, in this case into the effective valence band of water $\left(\mathrm{VB}_{\mathrm{H}_{2} \mathrm{O}}\right.$, see below $) .{ }^{18}$ This process is similar to the emission of electrons into the effective conduction band and occurs only under UV excitation. ${ }^{16,17} \mathrm{UV}$ radiation constitutes only $3 \%$ of the solar spectrum. If based only on this effect, our approach would not utilize the broadband optical absorption of npAu.

The photoemission of electrons is influenced by the SVR, as was identified earlier via the npAu approach. ${ }^{16}$ The question arises if a similar effect could be identified for the participation of hot holes to a reaction in the visible wavelength range. Figure 3B shows $\eta$ as a function of $1 / L$ (which is proportional to the SVR, see inset of Figure 1E) for different excitation wavelengths. From this plot, we observe an increase in $\eta$ with decreasing $L$ for all $\lambda$. This increase is found to be linear for highest $\lambda$ (i.e., for 708 and $802 \mathrm{~nm}$ illumination), with a tendency to form a plateau at smaller values of $L$ when $\lambda$ is decreased to $454 \mathrm{~nm}$. Below $454 \mathrm{~nm}$, this plateau again equalizes to give a straight line (at $368 \mathrm{~nm}$ ) and finally the flattening shifts toward larger $L$ for the values obtained under the lowest $\lambda$. Linear fits in Figure 3B (for all four points at 708 and $802 \mathrm{~nm}$, for the three largest $L$ values from 632 to $368 \mathrm{~nm}$ and for the two largest $L$ values for 335-307 nm) show a common root at $(46 \pm 2) \mathrm{nm}$, that is, the size at which, independent of $\lambda$, no more photoeffect can be expected. This, we believe, can be attributed to the transition from a regime where mainly electron-electron collisions in the bulk are responsible for damping to a regime where free electrons dominantly collide with the surface to transfer their momenta. As this speculation requires more detailed and accurate data, we would like to continue this discussion in a future contribution.

The electrochemical approach enables the investigation of surface reactions at different electrode potentials, $E$. As long as $E<1.35 \mathrm{~V}$, the CV (Figure 1E) shows no significant currents, and the SER spectra in Figure 1F show no chemical change of the surface. $I_{\mathrm{ph}}$ is determined under constant $E$, while eventual changes due to photovoltages ${ }^{70}$ are compensated by the potentiostat. ${ }^{16,70}$ From the plot at the bottom of Figure 3C, an increase in $\eta$ is identified when $E>1.3 \mathrm{~V}$, and a maximum for all wavelengths is found at $1.4 \mathrm{~V}$ before the photocurrent again drops for $E>1.4 \mathrm{~V}$. Note that npAu layers on fused silica showed a strong delamination from the substrate when $E>1.5$ $\mathrm{V}$ (fused silica substrates were used to guarantee a single optical transmission event through the layer and avoid 
reflection from an eventual metal substrate to lead to a second absorption) so that $E$ ranges that are relevant for OER could not be investigated. However, in Figure 3C, a correlation of the plot of $\eta v s$. E with the positive scan of the CV (middle plot) can be observed. Under the assumptions made for the oxidation of the npAu surface (Section SI-B), the coverage $\theta_{\mathrm{Au}-\mathrm{OH}}$ (upper plot in Figure $3 \mathrm{C}$ ) was calculated, from which the maximum in $\eta$ can be attributed to an $\mathrm{OH}$ coverage of $1 / 4$ of a monolayer $(=0.25$ monolayer $(\mathrm{ML}))$. It is important to note that these coincidences still are not capable of unequivocally revealing any causality between the photocurrent and the coverage.

2L-SERS. SERS enables the identification of near-surface (i.e. adsorbed or in very close proximity) chemical species by utilizing a near-field enhancement effect. ${ }^{59}$ For npAu electrodes with small feature sizes and high SVR, one can expect a high sensitivity since the screened surface will be larger than the geometrically observed surface. ${ }^{71}$ When combining the photoelectrochemical approach with in situ SERS, potential photochemical reaction products could be characterized directly. The in situ 2L-SERS method (see Methods section and section SI-F for details, andFigure SI-6 for the laser calibration) therefore utilizes a custom-made electrochemical in situ SERS cell (see Figure 4A) with the Raman laser focused onto the npAu electrode at the bottom of the cell. During the acquisition of a spectrum, a second laser is focused on the same spot to trigger the photochemical process. Under a static potential $E$, a product formed by the photoelectrochemical process can be characterized instantly via the Raman spectrum provided that it has Raman-active vibration modes, such as the $\mathrm{Au}-\mathrm{O}$ bond. From Figure $4 \mathrm{~B}$, we observe the broad $\mathrm{Au}-\mathrm{O}$ vibration peak in the range of $405-700 \mathrm{~cm}^{-1}$ which sets in between 1.3 and $1.4 \mathrm{~V}$ vs RHE in the spectra without illumination (thin lines, consistent with Figure $1 \mathrm{~F}$ ). When additionally illuminating the electrode with the $532 \mathrm{~nm}$ laser of larger spot size, we observe no relevant additional peaks (Figure 4B only shows the spectral range relevant for further discussion; outside of this range no further signals were observed). Note that Raman spectra can be considered representative for the entire sample since they were reproduced on multiple spots and from areas that are significantly larger than typical microstructural and erroneous features such as ligaments, cracks, or thickness variations, respectively. On the other hand, at $E=1.4 \mathrm{~V}$, we observe an increase in the scattering intensity (and a decrease back to the dark values when switching off illumination). The integral of SERS intensity is linearly dependent on the concentration of scattering species, that is, in this case, it is representative for $\theta_{\mathrm{Au}-\mathrm{OH}}{ }^{72}$ One can conclude that illumination causes the formation of additional $\mathrm{Au}-\mathrm{OH}$ species. To further strengthen this conclusion, we carried out a systematic study of the effect under variation of $E, P$, and $\lambda$. The results for selected potentials are shown in Figure 5. Spectra similar to Figure 4B for a non-annealed npAu sample can be found in Figure SI-7.

All spectra in Figure 5 were acquired under comparable conditions for all values of $\lambda$, so that the integrated counts within the given wavenumber range and hence the signal intensity can be compared quantitatively. Please note that the illuminated area among different lasers might be slightly different due to different spot sizes of different lasers; however, excitations occurring within these areas do not contribute to the integral values as the Raman laser spot size is smaller than that of the excitation laser. As a first observation, we again see

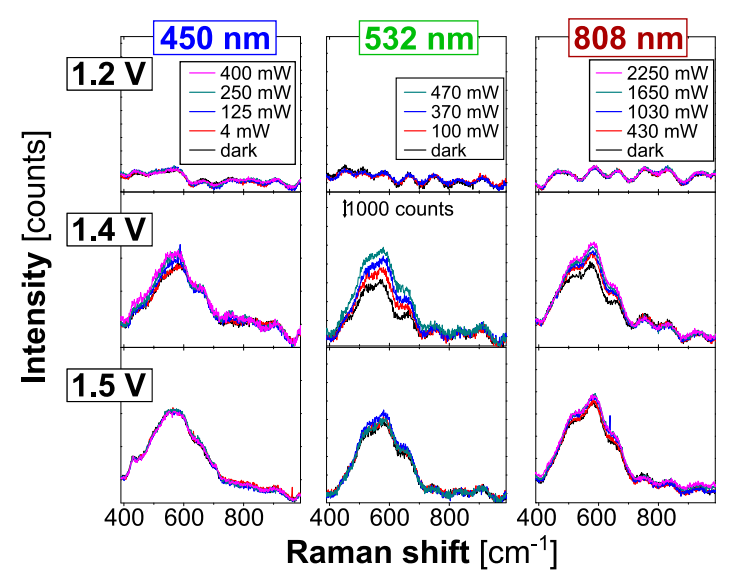

Figure 5. 2L-SER spectra measured at $\mathrm{npAu}$ with $L=11 \mathrm{~nm}(2 \mathrm{~min}$ annealing) under variation of the applied potential, the wavelength of the second laser, and its power (according to the calibration data in Figure SI-6).

no changes in signal intensity when increasing $P$ at $E<E_{\mathrm{ad} s}$ that is, before $\mathrm{Au}-\mathrm{OH}$ is formed under dark conditions (1.2 $\mathrm{V}$ ), which we regard as a further proof that photovoltages are compensated by the potentiostat. ${ }^{73}$ Second, we observe that the increase of the $\mathrm{Au}-\mathrm{OH}$ peak intensity with increased $P$ at $E=1.4 \mathrm{~V}$ depends on the wavelength of the second laser. Third, this behavior is not found after $E$ had been increased to $1.5 \mathrm{~V}$. At this potential, $\theta_{\mathrm{Au}-\mathrm{OH}}$ can be assumed $\approx 1$ (since $E_{\theta=1}$ $=1.44 \mathrm{~V}$ ), that is, there is no more free site available that can be oxidized by optically generated hot holes. As discussed in the Sample Characterization section, SER spectra are not capable of ruling out a further oxidation of the $\mathrm{Au}-\mathrm{OH}$ species to $\mathrm{Au}-\mathrm{O}$ (within 1.44-1.63 V), and results show that this is not connected to an increase in the total $\mathrm{O}$ content on the surface (spectra at $1.5 \mathrm{~V}$ ).

Figure 6A shows a summary of the second finding (the peak area increase at the formation potential of $\mathrm{Au}-\mathrm{OH}$ ) as represented by the peak integral determined within the 405$700 \mathrm{~cm}^{-1}$ spectral range as a function of $P$. From this plot, it is clearly visible that the obtained fits exhibit different slopes depending on the wavelength in the same way as those of the photocurrent in Figure 2C. When plotting the slope of the in situ 2L-SERS signal to $P$ vs. $E$ (Figure 6B), we see that, consistent with the sensitivity in Figure 3C, the signal intensity increase is highest, that is, the $\mathrm{Au}-\mathrm{OH}$ formation sensitivity is highest, between the onset potential $E_{\text {ads }}$ of surface oxidation and the subsequent peak potential (with $\theta_{\mathrm{Au}-\mathrm{OH}}=0.25$ ).

As every method that intends to show that hot charge carriers participate in the ongoing (surface) chemistry should be evaluated on whether it (exclusively) screens thermal effects, we checked the influence of an increased electrolyte temperature on the outcome of in situ SERS (no second laser). In this experiment, we assumed that the surrounding temperature will not be exceeded by a heating of the electrode due to photon absorption. Furthermore, we can neglect any thermal offset in the reference potential because the RHE used was located in a separate compartment which was at room temperature. Still, additional thermal potential differences between the npAu electrode and the RHE might occur, but these would similarly arise if plasmonic heating would be evident. As a sole difference to the preceding measurements, we note that the cell arrangement for the $T$ adjustability was different so that sample tilts (which sensitively result in SERS 

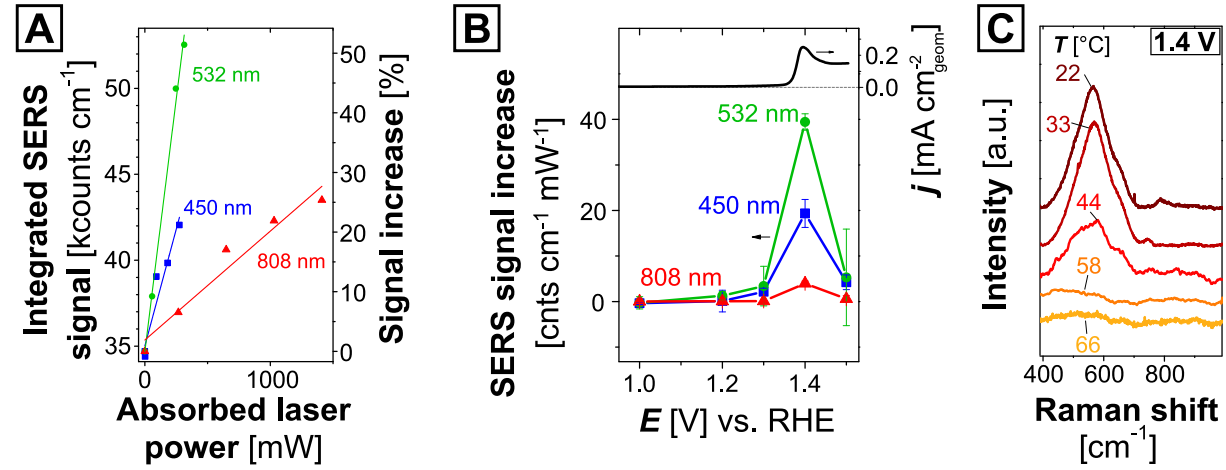

Figure 6. Results of $2 \mathrm{~L}-\mathrm{SERS}$ study on $\mathrm{npAu}$ with $L=11 \mathrm{~nm}(2 \mathrm{~min}$ annealing). (A) Au-O(H) peak integral at a potential $E=1.4 \mathrm{~V} v \mathrm{vs}$. RHE (within $405-700 \mathrm{~cm}^{-1}$ ) under illumination with different wavelengths of the second laser (indicated in the figure) vs. its power $P$ (normalized by the wavelength-specific value of absorption) including linear regression lines. (B) Slope of the linear regression lines in (A) (left axis) vs. $E$ (error bars result from the error in the slope as determined by linear regression in (A)) and comparison with the positive scan from CV (right axis, taken from Figure 1E). (C) SER spectra (no second laser) recorded at $E=1.4 \mathrm{~V}$ and varied static electrolyte temperatures.
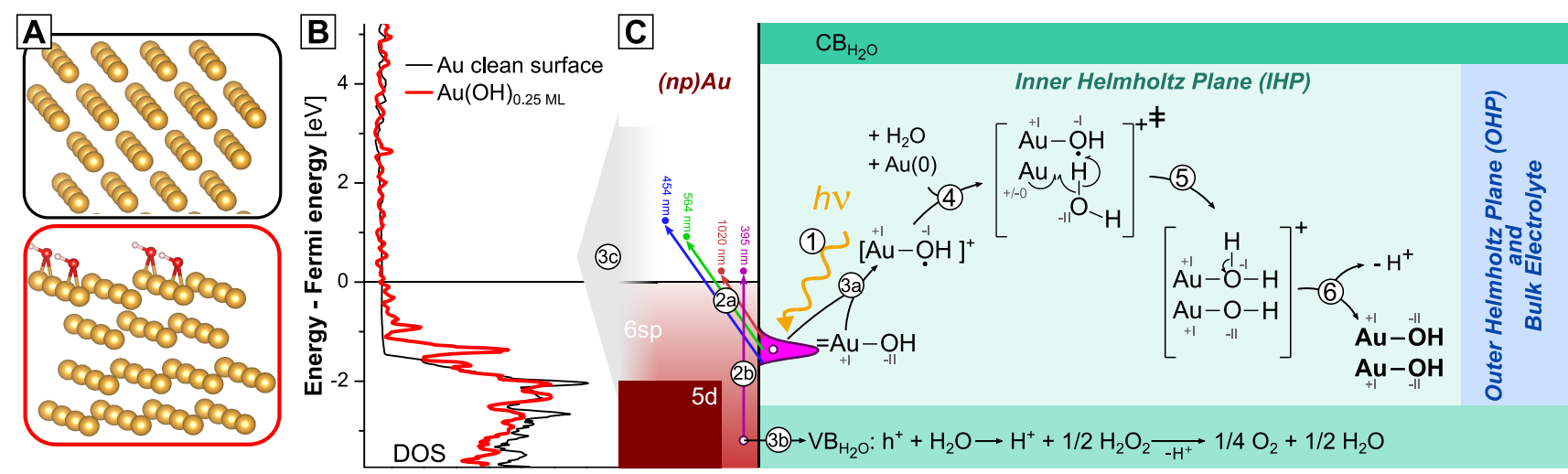

Figure 7. Mechanism of direct excitation and hot hole transfer from hybrid binding orbitals to (np)Au. (A) Assumed adsorbate geometries for the DFT calculation of the hybrid orbital energies (top: uncovered Au(111), bottom: $\mathrm{Au}(111)$ covered with $0.25 \mathrm{monolayer}$ (ML) hydroxide ( $\mathrm{OH}$ ) adsorbates). (B) Projected DOS for a clean (thin black) and OH-covered (bold red line) Au(111) surface from DFT calculation (states of one surface atom are shown). (C) Excitation and transfer mechanism in the presence of $0.25 \mathrm{ML} \mathrm{OH}$ adsorbates at 1.4 $\mathrm{V}$ (which results in a shift of the Fermi level with respect to Nernst potentials of adsorbate formation and bandgap of water). The hybridized orbital from DFT is shown by the idealized purple shape at the interface between (np)Au and the adjacent inner Helmholtz plane to the electrolyte (green background). The bandgap of water is given by its valence $\left(\mathrm{VB}_{\mathrm{H}_{2} \mathrm{O}}\right)$ and conduction band $\left(\mathrm{CB}_{\mathrm{H}_{2} \mathrm{O}}\right)$. See text for description of individual processes 1-6. Gray roman numbers represent oxidation numbers.

signal changes) could not be recognized, and hence the focusing procedure gave a different optimum aperture and exposure times leading to different noise levels, but not different peak shapes upon sample change. Figure 6C shows the SER spectra obtained under different electrolyte temperatures at $E=1.4 \mathrm{~V}$. In this figure, we observe that a temperature increase up to $66^{\circ} \mathrm{C}$ (a temperature previously identified to be sufficient to lead to significant catalytic rate increases) in general does not lead to an increase, but to a steady decrease in signal intensity. ${ }^{74}$ While the detailed investigation of this effect shall be subject to future contributions, we find that a $30 \mathrm{~K}$ temperature increase efficiently cancels the $\mathrm{Au}-\mathrm{OH}$ SERS signal. This, in contrast, is not observed (see Figures $4 \mathrm{~B}$ and 5) upon optical absorption of up to $2.2 \mathrm{~W}$ laser power, that is, we cannot conclude on a significant heating of the nanostructure by optical absorption.

Furthermore, we emphasize that our SERS measurements screen the static equilibrium adsorbate concentration, that is, the result of the adsorbate formation and decomposition equilibrium:

$$
\mathrm{Au}+\mathrm{H}_{2} \mathrm{O} \rightleftharpoons \mathrm{Au}-\mathrm{OH}+\mathrm{H}^{+}+\mathrm{e}^{-}
$$

This equilibrium and hence the concentration of $\mathrm{Au}-\mathrm{OH}$ is dependent on E. As 2L-SERS showed an increase in this concentration at a constant potential, we can conclude that illumination causes a shift of the equilibrium coverage which is independent of the applied potential.

Direct Charge-Transfer Mechanism. We find that the highest values for $\eta$ (Figure 3) and the most significant SERS signal increases with illumination occur at $\theta_{\mathrm{Au}-\mathrm{OH}} \approx 0.25$. Besides, we find a second peak for a SERS enhancement at 1.7 $\mathrm{V}$, that is, between the onset of multilayer $\mathrm{O}$ adsorption and OER, hence at an $\mathrm{O}$ coverage $\theta_{\mathrm{Au}-\mathrm{O}} \geq 1$. It is known that adsorbates bind to the surface via hybridized metal-adsorbate orbitals. $^{75}$ The energy of the hybrid-HOMO can be obtained from the density of states (DOS) of adsorbates on Au surfaces calculated within the framework of density functional theory (DFT, here using the software VASP.5.4.4, for details see the Methods section). ${ }^{76,77}$ Figure 7A shows simplified surface geometries (in a side view) of various surface geometries: a clean and adsorbate-free $\mathrm{Au}(111)$ surface (top), which we 
assume as a representative for our case and the same surface covered by $0.25 \mathrm{ML}$ of $\mathrm{OH}$ adsorbates in a bridge-like geometry (bottom). ${ }^{62}$ Note that the calculation for the adsorption geometry for a fully covered surface (as would be the case at $E>1.44 \mathrm{~V}$ ) requires the assumption of a different adsorption geometry, which we leave out of the scope of this manuscript for now since the most significant effects in the photocurrent signal are observed at lower coverages. DFT (Figure $7 \mathrm{~B}$ ) finds that a clean $\mathrm{Au}$ surface exhibits no states until the $5 \mathrm{~d}$-band onset at $-1.46 \mathrm{eV}$ (and a first peak at -2.05 $\mathrm{eV}$ ). With $0.25 \mathrm{ML}$ of $\mathrm{OH}$, we find additional states that evolve at $-1.15 \mathrm{eV}$ and $-0.9 \mathrm{eV}$.

Within the framework of DFT, the calculation and comparison of work functions of $\mathrm{Au}$ with and without surface adsorbates allows a quick derivation of the minimum onset energy. Calculating work functions usually requires testing of additional computational settings, for example, typically significantly more layers in the model systems are needed. An adequate derivation and discussion of work functions, for example, in dependence of the degree of adsorbate coverage exceeds both scope and extent of the present contribution, will therefore be supplied in detail in a follow-up contribution.

Taking the above observations on photocurrent and from 2L-SERS into account, we conclude on the reaction mechanism as displayed in Figure $7 \mathrm{C}$. At a potential of +1.4 $\mathrm{V}$, the electronic states of $\mathrm{Au}$ will be shifted by $1.4 \mathrm{eV}$ to more negative energies (together with the energies of adsorbatebinding orbitals) compared to the bandgap of water. ${ }^{78}$ This results in a shift of the Fermi level of $\mathrm{Au}$ (the horizontal black line in Figure $7 \mathrm{~B}, \mathrm{C}$ ) toward the valence band of water $\left(\mathrm{VB}_{\mathrm{H}_{2} \mathrm{O}}\right)$ and away from its conduction band $\left(\mathrm{CB}_{\mathrm{H}_{2} \mathrm{O}}\right)$. Figure $7 \mathrm{C}$ shows the situation after this shift and with $\theta_{\mathrm{Au}-\mathrm{OH}} \approx 0.25$, that is, where significant values of $\eta$ as well as an increase in reaction product concentration $(\mathrm{Au}-\mathrm{OH})$ are observed.

With adsorbates present, the hybrid-HOMO is filled and, as the linear combination of atomic orbitals (LCAO, see Section SI-G) reveals, has antibonding character, that is, can be denoted with $\mathrm{s} \pi_{\mathrm{y}}^{*}$ (see Figure SI-9). Analogous to other direct excitation mechanisms (as summarized, e.g., by Kazuma et al.), the absorption of a photon with energy $h \nu$ (process 1 in Figure 7C) excites an electron from this hybrid-HOMO (analog to an absorption under CID conditions), leaving behind localized holes in the surface state (process 2a). ${ }^{75,79}$ Withdrawing electrons from $\mathrm{s} \pi_{\mathrm{y}}^{*}$ would consequently lead to a strengthening of the $\mathrm{Au}-\mathrm{O}$ bond. In the LCAO scheme (Figure SI-9), these electrons represent free valence electrons of $\mathrm{OH}$ so that a withdrawal will create an unpaired valence electron, that is, a radical adsorbate species as shown after process 3 a. Note that Figure $7 \mathrm{C}$ shows only those excitation events that lead to a chemical reaction.

Upon wavelength decrease from 1020 to $600 \mathrm{~nm}$, we find an increase in $\eta$. According to Figure $7 \mathrm{C}$, the excitation from the hybrid state elevates electrons above the Fermi level after they have absorbed at least $1.15 \mathrm{eV}$. The dark red arrow in Figure $7 \mathrm{C}$ corresponds to the photon energy of $1.22 \mathrm{eV}(\lambda=1020$ $\mathrm{nm}$, the minimum that was available with our source) that creates reactive holes in the adsorbate state and hence a noticeable photocurrent since the excited electrons are extracted (process 3c). The value of $1.15 \mathrm{eV}$ was derived from the minimum energy of the hybrid HOMO as calculated by DFT. Note that an inhomogeneous broadening of the hybrid-HOMO state due to different orientations and defects on the $\mathrm{Au}$ surface might explain a smeared onset of $\eta$. Decreasing $\lambda$ causes an increased number of holes in the hybrid state according to its energetic distribution so that $\eta$ increases steadily until $564 \mathrm{~nm}(2.20 \mathrm{eV}$, green arrow). One would expect an edge-like characteristic originating from hybrid-state hot electrons as soon as they can be injected above the Fermi level. At approximately $2.2 \mathrm{eV}$, the absorption from the d-band starts to contribute more to absorption than that of free electrons and generates electrons above the Fermi level and holes in the d-band. ${ }^{80}$ While these still contribute to absorption, they cannot contribute to the reaction due to their strong localization, and as $\eta$ represents a normalization of the photocurrent (which decreases) to the absorption (which remains large), $\eta$ decreases and concludes a peak.

Simultaneous to process $2 \mathrm{a}$, free electrons in ( $\mathrm{np}$ ) Au can be excited to create hot carriers within (and not on the surface of) the metal (process 2b). So-formed hot holes will have a lifetime which is similarly short as that of hot electrons and do not lead to chemical reactions at the surface. If the photon energy is sufficiently high, 6sp electrons which are at the energy of the $\mathrm{VB}_{\mathrm{H}_{2} \mathrm{O}}$ can be excited (process $2 \mathrm{~b}$ ) to give holes that are emitted into water to contribute to its oxidation in a solution-based process. ${ }^{18}$ In contrast to these free holes, holes in the d-band will be formed, but due to their very low mobility, ${ }^{7}$ their contribution to the reaction at the surface can be neglected. However, we conclude that excess electrons which were formed by either mechanism ( $2 a$ and $2 b$ ) and which leave behind holes that participate in a reaction give a measurable photocurrent (process 3c).

At $\theta_{\mathrm{Au}-\mathrm{OH}}<1$, it is likely that an $\mathrm{OH}$ adsorbate is surrounded by a non-oxidized and uncovered $\mathrm{Au}$ atom $(\mathrm{Au}(0))$. When adding a $\mathrm{H}_{2} \mathrm{O}$ molecule which is the dominant species within the inner Helmholtz plane (IHP), an intermediate state forms via process 4 . $\mathrm{Au}(0)$ donates one electron to form a bond to the $\mathrm{O}$ atom of $\mathrm{H}_{2} \mathrm{O}$ so that $\mathrm{Au}(0)$ is oxidized to $\mathrm{Au}(\mathrm{I})$, the water molecule loses one $\mathrm{H}$ atom to form an adsorbed water molecule with the formerly adsorbed $\mathrm{OH}$ group and a second adsorbed $\mathrm{OH}$ group forms on the formerly non-covered $\mathrm{Au}(0)$ (process 5). Still, this state represents an energetically unfavorable cationic species which will further deprotonate (process 6) to finally form two neighboring $\mathrm{Au}-\mathrm{OH}$ species. Upon absorption of one photon that creates one hot hole $\mathrm{h}^{+}$inside the hybrid-HOMO, this entire process thus creates an additional, excess $(\mathrm{Au}-\mathrm{OH})_{\text {exc }}$ (with respect to the E-dependent equilibrium coverage) adsorbate species under the utilization of an existing $\mathrm{Au}-$ $\mathrm{OH}$ adsorbate $\left.(\mathrm{Au}-\mathrm{OH})_{\text {ads }}\right)$ to give a photocurrent according to the net equation:

$$
\mathrm{Au}(0)+\mathrm{H}_{2} \mathrm{O} \stackrel{(\mathrm{Au}-\mathrm{OH})_{\mathrm{ads}}}{\longrightarrow}(\mathrm{Au}-\mathrm{OH})_{\mathrm{exc}}+\mathrm{H}^{+}+\mathrm{e}^{-}
$$

which gives a photocurrent.

Figure $7 \mathrm{C}$ focuses on the participation of hot holes formed on the photoanode. There we observe continuous dark and photocurrents which imply a steadily ongoing $\mathrm{OH}$ adsorption reaction (at $E=1.4 \mathrm{~V}$, i.e., at $E<E_{\theta=1}$, compared with the current transients in Figure 2A). Over time this would cause a full oxidation of the npAu photoanode, followed by the formation of a multilayer oxide, that is, dissolution of the electrode. As $E<E_{\text {multi }}$, we do not observe decomposition even over prolonged durations. Consequently, the npAu surface is obviously not coated with a thicker oxide layer (SERS intensity for $\mathrm{Au}-\mathrm{O}$ under both dark and illuminated conditions over 
time is constant). Instead, all $(\mathrm{Au}-\mathrm{OH})$ species that are produced (via the dark as well as the direct mechanism) and that exceed the potential-dependent equilibrium coverage (as defined by $E$ and the illumination parameters) must simultaneously decompose to $\mathrm{O}_{2}$. Since there was no observable gas formation at the photoanode surface, we conclude that the amount of $\mathrm{O}_{2}$ generated within the observed time is too low to exceed its solubility in water $\left(8 \mathrm{mg} \mathrm{L}^{-1}\right)$. Note that further experiments with reduced pressure and a gas chromatography quantification of $\mathrm{O}_{2}$ are required to confirm this hypothesis.

Consequently and as indicated by the various excitation wavelengths in Figure $7 \mathrm{C}$, the process of hot hole-enhanced adsorbate formation is, as observed, a true broadband effect that happens at photon energies equal to the $s \pi_{\mathrm{y}}^{*}$ energy, that is, is active under NIR wavelengths and with this can be concluded to be advantageous over approaches that utilize narrow wavelength ranges of significant absorption, for example, by plasmon resonance.

\section{CONCLUSION}

Using of the full visible spectrum for plasmon-enhanced water splitting has been demonstrated by a photoelectrochemical approach with nanoporous gold as a single active light absorbing and water oxidizing thin film electrode. At potentials coinciding with the onset of surface oxidation, we observed a significant jump-like current with illumination. Their height (denoted as photocurrent) was linearly proportional to the incident power over all visible wavelengths under investigation. Thereby, the dependence of the photocurrent on the illuminating wavelength shows a non-monotonous behavior with the highest sensitivity values at green wavelengths. Similarly, the internal quantum efficiency of the process showed a peak at these wavelengths due to a transition in the dominant absorption from sp-band electrons (which generate reactive holes at large wavelengths) to d-band electrons (which increasingly absorb light at shorter wavelengths, but generate nonreactive holes) and finally to hot hole emission into water at UV wavelengths. The variation of the surface-to-volume ratio of the electrode revealed an exclusively surface-dominated absorption process as long as features are kept smaller than 46 $\mathrm{nm}$. Significant photocurrent values were found only at potentials at which adsorbates are stable. Within a modified surface-enhanced Raman spectroscopy approach, chemical changes of the surface as a consequence of illumination were monitored in situ. It revealed that illumination causes an increased equilibrium coverage of $\mathrm{Au}-\mathrm{OH}$. In this respect, we found the same qualitative dependence on potential, power, and wavelength, as identified in the photocurrent measurement. Density functional theory calculations showed an additional energetic state which was found to be the highest occupied hybrid molecular orbital of $\mathrm{OH}$ on $\mathrm{Au}$. From this state, electrons are directly transferred into states above the metal Fermi level so that longer-lived and hence highly reactive holes remain. These bind water to increase the already existing $\mathrm{OH}$ coverage on $\mathrm{Au}$. With this, the coverage can be concluded to strictly affect photon absorption and a direct excitationtransfer process which had been predicted, but yet not experimentally verified. Although exhibiting comparably low internal quantum efficiencies, the approach revealed its potential in strong current increases and its broadband nature.

\section{METHODS}

npAu Synthesis. Nanoporous Au photelectrodes were fabricated as reported previously. ${ }^{16}$ Briefly, white gold sheets $\left(\mathrm{Au}_{25} \mathrm{Ag}_{75}\right.$, Eytzinger Germany) of $100 \mathrm{~nm}$ nominal thickness were dealloyed while swimming on a $1 \mathrm{M} \mathrm{HClO}_{4}$ (70\% p.a., Merck, Germany) electrolyte solution being contacted as the working electrode (WE) via a $\mathrm{Au}$ wire from the top. A two-step potentiostatic dealloying protocol of $60 \mathrm{~min}+0.837 \mathrm{~V}$ vs. a $\mathrm{Ag} / \mathrm{AgCl} 3 \mathrm{M} \mathrm{KCl}$ (Metrohm Autolab Germany) commercial reference electrode (RE) and a polished $\mathrm{Ag}$ wire counter electrode (CE) and $+0.937 \mathrm{~V}$ for $15 \mathrm{~min}$ lead to the formation of self-supporting npAu sheets swimming on the electrolyte solution. These were cleaned by carefully exchanging the used electrolyte solution by deionized water with a syringe. The addition of a few drops of ethanol after 5 min enabled the quick and crack-free (see Figure SI-1) stripping of these layers onto the used substrate. For absorption and photocurrent measurements, these were acetone-cleaned fused silica microscope slides (Ted Pella, USA, cut to $2.5 \times 2.5 \mathrm{~cm}^{2}$ ) which were functionalized with an organic monolayer of 3-mercaptopropyltrimethoxysilane (MPTMS) by keeping the substrate in a $\mathrm{N}_{2}$-bubbled $30 \mathrm{mM}$ solution of MPTMS in toluene for $60 \mathrm{~min}$. After stripping, the layers were dried using a commercial fan and stored under air. Annealing was performed in a lab oven operated at $300{ }^{\circ} \mathrm{C}$ for varied durations $2-8 \mathrm{~min}$.

Sample Characterization. Scanning electron microscopy (SEM) was done in a Leo Gemini 1530 microscope equipped with an energydispersive X-ray (EDX) detector by Oxford Instruments. The average ligament diameter $L$ was estimated based on top-view images at $250,000 \times$ magnification by averaging a number of 25 ligament widths crossed by an imaginary image diagonal. ${ }^{61} \mathrm{Ag}$ content was determined by EDX from sample areas of $20 \times 30 \mu \mathrm{m}$ in size.

For electrochemical, SERS, and 2L-SERS measurements, npAu was stripped onto a mirror-polished Au substrate (polishing with 3, 1, 0.25 , and $0.05 \mu \mathrm{m}$ for $2 \mathrm{~min}$ each using a diamond polishing paste over a polishing gauze both by Buehler, Germany, cleaning for $3 \times 2$ min in water under ultrasonic agitation). The cells for all electrochemical, SERS, and 2L-SERS characterization were cleaned according to the following procedure: Overnight immersion of all glassware into a $\mathrm{KMnO}_{4} / \mathrm{H}_{2} \mathrm{SO}_{4} / \mathrm{H}_{2} \mathrm{O}_{2}$ solution, followed by four times cleaning in deionized water and four times boiling in deionized water. The sample carried on the massive Au was characterized with a $\mu$ Autolab potentiostat by Metrohm, Netherlands, after overnight immersion into an $\operatorname{Ar}(5.0)$-flushed $0.5 \mathrm{M} \mathrm{H}_{2} \mathrm{SO}_{4}$ (Sigma-Aldrich, Suprapure) electrolyte. All potentials refer to a Pt wire RHE (flushed by $5.0 \mathrm{H}_{2}$ ). The $\mathrm{CE}$ used was a flame-annealed Au wire. $\mathrm{CVs}$ were recorded at varied scan rate (see Figure SI-2A) showing the typical features of $\mathrm{Au}$ oxidation and reduction.

Optical absorption spectra of the samples were derived using a spectrometer (PerkinElmer Lambda 1050). Similar to the samples for photocurrent measurement, $\mathrm{npAu}$ samples for spectroscopic characterization were cast onto fused silica substrates in order to prevent UV absorption of glass. After drying, samples were immersed into a $0.5 \mathrm{M}$ $\mathrm{H}_{2} \mathrm{SO}_{4}$ electrolyte to soak, while excess liquid was removed before spectroscopy. Absorption spectra were determined by subtracting transmission, reflection, and scattering (measured using an integrating sphere setup) contributions from $100 \%$. Spectra of the optical absorption $A$ were taken from earlier results and are displayed in Figure SI-4. ${ }^{16}$

Photocurrent measurements were performed as described priorly, that is, npAu layers on fused silica substrates were installed as WE in a photoelectrochemical cell by Zahner, Germany, exposing a circular area of $1.8 \mathrm{~cm}$ diameter $\left(2.54 \mathrm{~cm}^{2}\right)$ geometric area to the illumination of a wavelength and power-tunable light source TLSO3 (emission spectrum see Figure SI-5) while being in contact with a freshly degassed $0.5 \mathrm{M} \mathrm{H}_{2} \mathrm{SO}_{4}$ (Alfa, Germany) electrolyte. ${ }^{16}$ In this cell, the $\mathrm{CE}$ was a polished $\mathrm{Pt}$ wire and the $\mathrm{RE}$ a $\mathrm{Ag} \mid \mathrm{AgCl}$ reference electrode by BASi, USA, with an offset of $-0.409 \mathrm{~V}$ to RHE. The entire setup was shielded in a dark box and controlled externally by a Zahner ZenniumX potentiostat. By the design of the cell, the diameter of the illuminated area does not exceed the exposed circular sample area. 
The photocurrents were determined at different constant potentials $E$ under varied illumination conditions (wavelength $\lambda$ and power $P$ ). After a constant current under dark conditions was established, the light was switched on and off for $5 \mathrm{~s}$, respectively, and photocurrents were determined from the jumps in the WE current by subtracting the current in the dark from that under illumination in the moment of switching by using linearly fitted current transients. This was done for five pulses in series so that average photocurrents $I_{\mathrm{ph}}$ could be determined including a standard deviation from which we regard as its standard error. $I_{\mathrm{ph}}$ was related to the absorbed power $P_{\mathrm{abs}}=P A$ and the photon energy $E_{\mathrm{ph}}=\hbar c / \lambda$ ( $\hbar$ is Planck's constant, $c$ is the speed of light in vacuum) via the internal quantum efficiency $\eta=I_{\mathrm{ph}} E_{\mathrm{ph}} / e P_{\mathrm{abs}}(e$ is the elementary charge). The determined $\eta$ values as shown in Figure $3 \mathrm{~A}$ are compared on an energy scale with the spectrum of the density of states (DOS) as determined via density functional theory (see DFT section). This comparison is displayed in Figure SI-6 for all ligament diameters $L$.

In Situ SERS. In situ SERS measurements were performed in a homemade electrochemical cell (see Figure 5A) similar to the above electrochemical cell, but equipped with a quartz window for the upside-down microscope geometry of the Horiba Yobin Yvon LabRAM HR. The sample was immersed in the $0.5 \mathrm{M} \mathrm{H}_{2} \mathrm{SO}_{4}$ electrolyte (Sigma-Aldrich, Suprapure) and slightly pressed against the quartz window. The details of the setup can be found elsewhere. ${ }^{81}$ The $632.8 \mathrm{~nm} 20 \mathrm{~mW}$ Raman laser was focused on the sample surface via a $50 \times$ lens by maximizing the Raman signal intensity in a real-time measurement.

2L-SERS. For 2L-SERS, an additional laser (compared to the priorly described SERS measurement) was directed to the identical spot on the sample as used for the Raman measurement, and its power was controlled externally. The individual calibration curves of the used lasers can be taken from Figure SI-7A, according to which the laser power was ramped up stepwisely. The lasers were all made by Civil Laser, China. For 450 and 532 nm lasers, we used a long-pass filter with $570 \mathrm{~nm}$ cutoff wavelength as a filter in front of the spectroscope, and for the $808 \mathrm{~nm}$ laser, we used a $700 \mathrm{~nm}$ short-pass filter, both by Thorlabs. The use of both filters resulted in no signal intensity loss in the relevant SERS wavelength range $(640-690 \mathrm{~nm})$, as determined using a $\mathrm{Si}$ calibration sample, that is, throughout the entire power spectrum of the second laser, no leaking laser intensity reached the detector of the spectroscope as shown by Figure SI-7B (signals in there were collected using a $\mathrm{Si}$ test sample so that the typical Raman features of $\mathrm{Si}$ are visible). The only detectable effect is found from the filter used in the system, namely a slight broadband reduction of the Raman intensity due to laser reflection on the glass filter. All SERS and 2L-SERS spectra were recorded under the same conditions, that is, the same aperture (D0.3), same exposure time (60 s), and same number of measurement repetitions (three). In $2 \mathrm{~L}-$ SERS, excitation with different wavelengths was achieved by exchanging the laser source and not shifting the measurement spot. After baseline subtraction using the software LabRam by Horiba Yobin Yvon, the Raman signal intensity was determined by automated integration (using the start and end points as a basis for an integration baseline) of the peak count values in the range $400-700 \mathrm{~cm}^{-1}$ using Origin. 2L-SER spectra of as-dealloyed npAu illuminated by a $532 \mathrm{~nm}$ laser compared to the respective spectra without illumination are shown in Figure SI-8.

DFT Calculations. Spin-polarized density functional theory (DFT) calculations were performed on atomic oxygen and $\mathrm{OH}$ groups adsorbed on $\mathrm{Au}(111)$ slabs as a simplified model system for npAu. The $\mathrm{Au}(111)$ was chosen because of its high thermodynamic stability. We are aware of the broad surface facet distribution in $\mathrm{npAu}$ which could lead to slight shifts in the resulting hybrid-HOMO energy, but would like to limit the discussion here to the simplest surface orientation.

In an experimental situation, we anticipate an inhomogeneous broadening of the HOMO energy state when the adsorbate binds to surfaces of different crystal plane orientations and defects on the surface. The calculations were done using the plane-wave code Vienna $a b$ Initio Simulation Package VASP.5.4.4. ${ }^{7,82}$ The projector augmented-wave method and periodic boundary conditions were employed in the calculations. ${ }^{77,83}$ The exchange-correlation energy contributions were calculated in the generalized gradient approximation using the Perdew, Burke, and Ernzerhof parametrization. ${ }^{84}$ An energy cutoff of $550 \mathrm{eV}$ and a $k$-point grid of $15 \times 15 \times 1$ for a $2 \times$ 2 surface cell were chosen, allowing for a convergence within $1 \mathrm{meV} /$ atom for the system described below. DOS were calculated by a static run using the tetrahedron method with Blöchl corrections following the structural relaxations. ${ }^{85}$ The adsorption was modeled by symmetric Au slabs with a periodic $2 \times 2$ surface cell and consisting of 12 layers. The number of layers was chosen to converge the DOS of the bulk-like central layers with respect to a bulk DOS. Perpendicular to the surface, the slabs are decoupled by a vacuum region of about $18 \AA$. The central three Au layers are kept fixed with bulk-like distances, whereas the surface layers are allowed to relax until the forces acting on them became smaller than $5 \mathrm{meV} / \mathrm{A}$. One oxygen atom or $\mathrm{OH}$ group was placed per $2 \times 2$ surface cell symmetrically on both sides of the slab, resulting in a coverage of 0.25 monolayers. At that coverage, a surface adsorption of an oxygen atom and an $\mathrm{OH}$ group at the fcc hollow site and a bridge site, respectively, is energetically most favorable. ${ }^{86}$ However, it has to be noted that coverages above $0.25 \mathrm{ML}$ may result in the occupation of mixed adsorption sites, the formation of surface oxides, and the incorporation into the Au structure. ${ }^{87,88}$

\section{ASSOCIATED CONTENT}

\section{SI Supporting Information}

The Supporting Information is available free of charge at https://pubs.acs.org/doi/10.1021/acsnano.0c09776.

Material characterization results by SEM, EDX and ECSA measurement, results of CV, details of surface oxidation process, SERS and 2L-SERS spectra for $n p A u$ without thermal treatment, absorption curves from UVvis spectroscopy, emission data of light source, spectra of internal quantum efficiency in comparison to DFT results, calibration data for lasers used for 2L-SERS, Raman spectrum of Si standard with and without second illumination, 2L-SERS spectra for npAu sample without thermal treatment, remarks for DFT calculations, LCAO scheme for $\mathrm{OH}$ binding to $\mathrm{Au}$, and references (PDF)

\section{AUTHOR INFORMATION}

\section{Corresponding Author}

Matthias Graf - Institute for Materials Research, Helmholtz Center Geesthacht, D-21502 Geesthacht, Germany; Leiden Institute of Chemistry, Leiden University, 2333 CD Leiden, The Netherlands; 10 orcid.org/0000-0002-2065-0014; Email: matthias.graf@tuhh.de

\section{Authors}

Gregor B. Vonbun-Feldbauer - Institute of Advanced Ceramics, Hamburg University of Technology, D-21703 Hamburg, Germany; 이이.org/0000-0002-9327-0450

Marc T. M. Koper - Leiden Institute of Chemistry, Leiden University, 2333 CD Leiden, The Netherlands; 10 orcid.org/ 0000-0001-6777-4594

Complete contact information is available at: https://pubs.acs.org/10.1021/acsnano.0c09776

\section{Notes}

The authors declare no competing financial interest. 


\section{ACKNOWLEDGMENTS}

The authors thank Diana Aranzales Ochoa from Universiteit Leiden for assistance with in situ SERS spectroscopy. This work was funded by the Deutsche Forschungsgemeinschaft (DFG, German Research Foundation) - project number 192346071 - SFB 986 and subprojects A4, B10, and C9.

\section{REFERENCES}

(1) Joya, K. S.; Joya, Y. F.; Ocakoglu, K.; Van De Krol, R. WaterSplitting Catalysis and Solar Fuel Devices: Artificial Leaves on the Move. Angew. Chem., Int. Ed. 2013, 52 (40), 10426-10437.

(2) Stolarczyk, J. K.; Bhattacharyya, S.; Polavarapu, L.; Feldmann, J. Challenges and Prospects in Solar Water Splitting and $\mathrm{CO} 2$ Reduction with Inorganic and Hybrid Nanostructures. ACS Catal. 2018, 8, 3602-3635.

(3) Fujishima, A.; Honda, K. Electrochemical Photolysis of Water at a Semiconductor Electrode. Nature 1972, 238 (5358), 37-38.

(4) Linic, S.; Christopher, P.; Ingram, D. B. Plasmonic-Metal Nanostructures for Efficient Conversion of Solar to Chemical Energy. Nat. Mater. 2011, 10, 911-921.

(5) Warren, S. C.; Thimsen, E. Plasmonic Solar Water Splitting. Energy Environ. Sci. 2012, 5 (1), 5133-5146.

(6) Doyle, R. L.; Lyons, M. E. G. The Mechanism of Oxygen Evolution at Superactivated Gold Electrodes in Aqueous Alkaline Solution. J. Solid State Electrochem. 2014, 18 (12), 3271-3286.

(7) Khurgin, J. B. Hot Carriers Generated by Plasmons: Where Are They Are Generated and Where Do They Go from There? Faraday Discuss. 2019, 214, 35-58.

(8) Uskov, A. V.; Khurgin, J. B.; Protsenko, I. E.; Smetanin, I. V.; Bouhelier, A. Excitation of Plasmonic Nanoantennas by Nonresonant and Resonant Electron Tunnelling. Nanoscale 2016, 8, 14573-14579.

(9) Manjavacas, A.; Liu, J. G.; Kulkarni, V.; Nordlander, P. PlasmonInduced Hot Carriers in Metallic Nanoparticles. ACS Nano 2014, 8 (8), 7630-7638.

(10) Rodio, M.; Graf, M.; Schulz, F.; Mueller, N. S.; Eich, M.; Lange, H. Experimental Evidence for Nonthermal Contributions to PlasmonEnhanced Electrochemical Oxidation Reactions. ACS Catal. 2020, 10 (3), 2345-2353.

(11) Khurgin, J. B. Fundamental Limits of Hot Carrier Injection from Metal in Nanoplasmonics. Nanophotonics 2020, 9 (2), 453-471.

(12) Blandre, E.; Jalas, D.; Petrov, A. Y.; Eich, M. Limit of Efficiency of Generation of Hot Electrons in Metals and Their Injection inside a Semiconductor Using a Semi-Classical Approach. ACS Photonics 2018, 5, 3613-3620.

(13) Sivan, Y.; Un, I. W.; Dubi, Y. Assistance of Metal Nanoparticles in Photocatalysis - Nothing More Than a Classical Heat Source. Faraday Discuss. 2019, 214, 215-233.

(14) Zhou, L.; Swearer, D. F.; Zhang, C.; Robatjazi, H.; Zhao, H.; Henderson, L.; Dong, L.; Christopher, P.; Carter, E. A.; Nordlander, P.; Halas, N. Quantifying Hot Carrier and Thermal Contributions in Plasmonic Photocatalysis. Science 2018, 362 (6410), 69-72.

(15) Xie, W.; Schlücker, S. Hot Electron-Induced Reduction of Small Molecules on Photorecycling Metal Surfaces. Nat. Commun. 2015, 6, 7570-7575.

(16) Graf, M.; Jalas, D.; Weissmüller, J.; Petrov, A. Y.; Eich, M. Surface-to-Volume Ratio Drives Photoelelectron Injection From Nanoscale Gold into Electrolyte. ACS Catal. 2019, 9, 3366-3374.

(17) Brodsky, A. M.; Pleskov, Y. V. Electron Photoemission at a Metal-Electrolyte Solution Interface. Prog. Surf. Sci. 1972, 2, 1-73.

(18) Watanabe, T.; Gerischer, H. Photoelectrochemical Studies on Gold Electrodes with Surface Oxide Layers - Part II. Discrimination between the Surface Photoprocess and Photohole Emission. J. Electroanal. Chem. Interfacial Electrochem. 1981, 122, 73-91.

(19) Cortes, E.; Xie, W.; Cambiasso, J.; Jermyn, A. S.; Sundararaman, R.; Narang, P.; Schlucker, S.; Maier, S. A. Plasmonic Hot Electron Transport Drives Nano-Localized Chemistry. Nat. Commun. 2017, 8, 14880.
(20) Wu, X.; Thrall, E. S.; Liu, H.; Steigerwald, M.; Brus, L. Plasmon Induced Photovoltage and Charge Separation in Citrate-Stabilized Gold Nanoparticles. J. Phys. Chem. C 2010, 114, 12896-12899.

(21) Linic, S.; Aslam, U.; Boerigter, C.; Morabito, M. Photochemical Transformations on Plasmonic Metal Nanoparticles. Nat. Mater. 2015, 14 (6), 567-576.

(22) Carneiro-Neto, E. B.; Lopes, M. C.; Pereira, E. C. Simulation of Interfacial $\mathrm{PH}$ Changes during Hydrogen Evolution Reaction. J. Electroanal. Chem. 2016, 765, 92-99.

(23) Bard, A. J.; Faulkner, L. R. Electrochemical Methods, 2nd ed.; John Wiley \& Sons Inc.: New York, 2001; pp 100-104.

(24) Wang, C.; Nie, X.-G.; Shi, Y.; Zhou, Y.; Xu, J.-J.; Xia, X.-H.; Chen, H.-Y. Direct Plasmon-Accelerated Electrochemical Reaction on Gold Nanoparticles. ACS Nano 2017, 11, 5897-5905.

(25) Reineck, P.; Brick, D.; Mulvaney, P.; Bach, U. Plasmonic Hot Electron Solar Cells: The Effect of Nanoparticle Size on Quantum Efficiency. J. Phys. Chem. Lett. 2016, 7 (20), 4137-4141.

(26) Tagliabue, G.; Jermyn, A. S.; Sundararaman, R.; Welch, A. J.; DuChene, J. S.; Pala, R.; Davoyan, A. R.; Narang, P.; Atwater, H. A. Quantifying the Role of Surface Plasmon Excitation and Hot Carrier Transport in Plasmonic Devices. Nat. Commun. 2018, 9, 3394.

(27) Mubeen, S.; Lee, J.; Singh, N.; Krämer, S.; Stucky, G. D.; Moskovits, M. An Autonomous Photosynthetic Device in Which All Charge Carriers Derive from Surface Plasmons. Nat. Nanotechnol. 2013, 8 (4), 247-251.

(28) Wu, K.; Chen, J.; McBride, J. R.; Lian, T. Efficient Hot-Electron Transfer by a Plasmon-Induced Interfacial Charge-Transfer Transition. Science (Washington, DC, U. S.) 2015, 349, 632-635.

(29) Ratchford, D. C.; Dunkelberger, A. D.; Vurgaftman, I.; Owrutsky, J. C.; Pehrsson, P. E. Quantification of Efficient Plasmonic Hot-Electron Injection in Gold Nanoparticle-TiO2 Films. Nano Lett. 2017, 17, 6047-6055.

(30) Lewis, N. An Analysis of Charge Transfer Rate Constants for Semiconductor/Liquid Interfaces. Annu. Rev. Phys. Chem. 1991, 42 (1), 543-580.

(31) Foerster, B.; Joplin, A.; Kaefer, K.; Celiksoy, S.; Link, S.; Sönnichsen, C. Chemical Interface Damping Depends on Electrons Reaching the Surface. ACS Nano 2017, 11, 2886-2893.

(32) Kale, M. J.; Avanesian, T.; Christopher, P. Direct Photocatalysis by Plasmonic Nanostructures. ACS Catal. 2014, 4 (1), 116-128.

(33) Therrien, A. J.; Kale, M. J.; Yuan, L.; Zhang, C.; Halas, N. J.; Christopher, P. Impact of Chemical Interface Damping on Surface Plasmon Dephasing. Faraday Discuss. 2019, 214, 59-72.

(34) Kreibig, U. Interface-Induced Dephasing of Mie Plasmon Polaritons(1) Kreibig, U. Interface-Induced Dephasing of Mie Plasmon Polaritons. Appl. Phys. B: Lasers Opt. 2008, 93 (1), 7989; Appl. Phys. B: Lasers Opt. 2008, 93, 79-89.

(35) Boerigter, C.; Campana, R.; Morabito, M.; Linic, S. Evidence and Implications of Direct Charge Excitation as the Dominant Mechanism in Plasmon-Mediated Photocatalysis. Nat. Commun. 2016, 7, 10545.

(36) Christopher, P.; Xin, H.; Marimuthu, A.; Linic, S. Singular Characteristics and Unique Chemical Bond Activation Mechanisms of Photocatalytic Reactions on Plasmonic Nanostructures. Nat. Mater. 2012, 11, 1044-1050.

(37) Foerster, B.; Hartelt, M.; Collins, S. S. E.; Aeschlimann, M.; Link, S.; Sönnichsen, C. Interfacial States Cause Equal Decay of Plasmons and Hot Electrons at Gold - Metal Oxide Interfaces Interfacial States Cause Equal Decay of Plasmons and Hot Electrons at Gold - Metal Oxide Interfaces. Nano Lett. 2020, 20 (5), 33383343.

(38) Kale, M. J.; Christopher, P. Plasmons at the Interface. Science (Washington, DC, U. S.) 2015, 349 (6248), 587-588.

(39) Kreibig, U.; Vollmer, M. Optical Properties of Metal Clusters; Springer: Berlin, 1995.

(40) Schlather, A. E.; Manjavacas, A.; Lauchner, A.; Marangoni, V. S.; DeSantis, C. J.; Nordlander, P.; Halas, N. J. Hot Hole Photoelectrochemistry on $\mathrm{Au} @ \mathrm{SiO} 2 @ \mathrm{Au}$ Nanoparticles. J. Phys. Chem. Lett. 2017, 8 (9), 2060-2067. 
(41) Mukherjee, S.; Zhou, L.; Goodman, A. M.; Large, N.; AyalaOrozco, C.; Zhang, Y.; Nordlander, P.; Halas, N. J. Hot-ElectronInduced Dissociation of $\mathrm{H} 2$ on Gold Nanoparticles Supported on SiO2. J. Am. Chem. Soc. 2014, 136 (1), 64-67.

(42) Duchene, J. S.; Tagliabue, G.; Welch, A. J.; Cheng, W. H.; Atwater, H. A. Hot Hole Collection and Photoelectrochemical CO2 Reduction with Plasmonic Au/p-GaN Photocathodes. Nano Lett. 2018, 18 (4), 2545-2550.

(43) Kim, H. J.; Lee, S. H.; Upadhye, A. A.; Ro, I.; Tejedor-Tejedor, M. I.; Anderson, M. A.; Kim, W. B.; Huber, G. W. Plasmon-Enhanced Photoelectrochemical Water Splitting with Size-Controllable Gold Nanodot Arrays. ACS Nano 2014, 8, 10756-10765.

(44) Weissmüller, J.; Newman, R. C.; Jin, H.; Hodge, A. M.; Kysar, J. W. Nanoporous Metals by Alloy Corrosion: Formation and Mechanical Properties. MRS Bull. 2009, 34, 577-586.

(45) Sieradzki, K.; Corderman, R. R.; Shukla, K.; Newman, R. C. Computer Simulations of Corrosion: Selective Dissolution of Binary Alloys. Philos. Mag. A 1989, 59 (4), 713-746.

(46) Hammer, B.; Norskov, J. K. Theoretical Surface Science and Catalysis - Calculations and Concepts. Adv. Catal. 2000, 45, 71129.

(47) Wittstock, A.; Wichmann, A.; Bäumer, M. Nanoporous Gold as a Platform for a Building Block Catalyst. ACS Catal. 2012, 2, 21992215.

(48) Krekeler, T.; Straßer, A. V.; Graf, M.; Wang, K.; Hartig, C.; Ritter, M.; Weissmüller, J. Silver-Rich Clusters in Nanoporous Gold. Mater. Res. Lett. 2017, 5, 314.

(49) Graf, M.; Haensch, M.; Carstens, J.; Wittstock, G.; Weissmüller, J. Electrocatalytic Methanol Oxidation with Nanoporous Gold: Microstructure and Selectivity. Nanoscale 2017, 9 (45), 1783917848.

(50) Steyskal, E.-M.; Seidl, M.; Graf, M.; Würschum, R. In Situ Resistometry Studies of the Dealloying Progress during Nanoporous Structure Evolution. Phys. Chem. Chem. Phys. 2017, 19, 2988029885.

(51) Smith, J. G.; Jain, P. K. The Ligand Shell as an Energy Barrier in Surface Reactions on Transition Metal Nanoparticles. J. Am. Chem. Soc. 2016, 138, 6765-6773.

(52) Comotti, M.; Della Pina, C.; Matarrese, R.; Rossi, M. The Catalytic Activity of "Naked" Gold Particles. Angew. Chem., Int. Ed. 2004, 43 (43), 5812-5815.

(53) Wang, Z.; Du, J.; Zhang, Y.; Han, J.; Huang, S.; Hirata, A.; Chen, M. Free-Standing Nanoporous Gold for Direct Plasmon Enhanced Electro-Oxidation of Alcohol Molecules. Nano Energy 2019, 56, 286-293.

(54) Parida, S.; Kramer, D.; Volkert, C. A.; Rösner, H.; Erlebacher, J.; Weissmüller, J. Volume Change during the Formation of Nanoporous Gold by Dealloying. Phys. Rev. Lett. 2006, 97 (3), 4-7.

(55) Diaz-Morales, O.; Calle-Vallejo, F.; de Munck, C.; Koper, M. T. M. Electrochemical Water Splitting by Gold: Evidence for an Oxide Decomposition Mechanism. Chem. Sci. 2013, 4 (6), 2334.

(56) Martina, I.; Wiesinger, R.; Schreiner, M. Micro-Raman Investigations of Early Stage Silver Corrosion Products Occurring in Sulfur Containing Atmospheres. J. Raman Spectrosc. 2013, 44, 770775 .

(57) Angerstein-Kozlowska, H.; Conway, B. E.; Hamelin, A.; Stoicoviciu, L. Elementary Steps of Electrochemical Oxidation of Single-Crystal Planes of Au-I. Chemical Basis of Processes Involving Geometry of Anions and the Electrode Surfaces. Electrochim. Acta 1986, 31 (8), 1051-1061.

(58) Yeo, B. S.; Klaus, S. L.; Ross, P. N.; Mathies, R. A.; Bell, A. T. Identification of Hydroperoxy Species as Reaction Intermediates in the Electrochemical Evolution of Oxygen on Gold. ChemPhysChem 2010, 11 (9), 1854-1857.

(59) Schlücker, S. Surface-Enhanced Raman Spectroscopy: Concepts and Chemical Applications. Angew. Chem., Int. Ed. 2014, 53, $4756-4795$.

(60) Zhang, Y.; Gao, X.; Weaver, M. J. Nature of Surface Bonding on Voltammetrically Oxidized Noble Metals in Aqueous Media as
Probed by Real-Time Surface-Enhanced Raman Spectroscopy. J. Phys. Chem. 1993, 97, 8656-8663.

(61) Graf, M.; Roschning, B.; Weissmüller, J. Nanoporous Gold by Alloy Corrosion: Method-Structure-Property Relationships. J. Electrochem. Soc. 2017, 164, C194-C200.

(62) Hoppe, S.; Li, Y.; Moskaleva, L. V.; Müller, S. How Silver Segregation Stabilizes 1D Surface Gold Oxide: A Cluster Expansion Study Combined with ab Initio MD Simulations. Phys. Chem. Chem. Phys. 2017, 19, 14845-14853.

(63) Moskaleva, L. V.; Röhe, S.; Wittstock, A.; Zielasek, V.; Klüner, T.; Neyman, K. M.; Bäumer, M. Silver Residues as a Possible Key to a Remarkable Oxidative Catalytic Activity of Nanoporous Gold. Phys. Chem. Chem. Phys. 2011, 13 (10), 4529-4539.

(64) Wittstock, A.; Bäumer, M. Catalysis by Unsupported Skeletal Gold Catalysts. Acc. Chem. Res. 2014, 47 (3), 731-739.

(65) Haensch, M.; Graf, M.; Wang, W.; Nefedov, A.; Wöll, C.; Weissmüller, J.; Wittstock, G. Thermally Driven Ag-Au Compositional Changes at the Ligament Surface in Nanoporous Gold: Implications for Electrocatalytic Applications. ACS Appl. Nano Mater. 2020, 3 (3), 2197-2206.

(66) Jalas, D.; Canchi, R.; Petrov, A. Y.; Lang, S.; Shao, L.; Weissmüller, J.; Eich, M. Effective Medium Model for the Spectral Properties of Nanoporous Gold in the Visible. Appl. Phys. Lett. 2014, 105,241906

(67) Arnob, M. M. P.; Zhao, F.; Zeng, J.; Santos, G. M.; Li, M.; Shih, W. C. Laser Rapid Thermal Annealing Enables Tunable Plasmonics in Nanoporous Gold Nanoparticles. Nanoscale 2014, 6 (21), 1247012475 .

(68) Zeng, J.; Zhao, F.; Qi, J.; Li, Y.; Li, C. H.; Yao, Y.; Lee, T. R.; Shih, W. C. Internal and External Morphology-Dependent Plasmonic Resonance in Monolithic Nanoporous Gold Nanoparticles. RSC Adv. 2014, 4 (69), 36682-36688.

(69) Ringe, E.; Zhang, J.; Langille, M.; Sohn, K.; Cobley, C.; Au, L.; Xia, Y.; Mirkin, C.; Huang, J.; Marks, L.; Duyne, R. Effect of Size, Shape, Composition, and Support Film on Localized Surface Plasmon Resonance Frequency: A Single Particle Approach Applied to Silver Bipyramids and Gold and Silver Nanocubes. MRS Proc. 2009, 1208, 1208-O10-02

(70) Redmond, P. L.; Wu, X.; Brus, L. Photovoltage and Photocatalyzed Growth in Citrate-Stabilized Colloidal Silver Nanocrystals. J. Phys. Chem. C 2007, 111, 8942-8947.

(71) Qian, L. H.; Yan, X. Q.; Fujita, T.; Inoue, A.; Chen, M. W. Surface Enhanced Raman Scattering of Nanoporous Gold: Smaller Pore Sizes Stronger Enhancements. Appl. Phys. Lett. 2007, 90 (15), 153120.

(72) Stolberg, L.; Lipkowski, J.; Irish, D. E. An Examination of the Relationship between Surface Enhanced Raman Scattering (SERS) Intensities and Surface Concentration for Pyridine Adsorbed at the Polycrystalline Gold/Aqueous Solution Interface. J. Electroanal. Chem. Interfacial Electrochem. 1991, 300, 563-584.

(73) Jalas, D.; Shao, L.-H.; Canchi, R.; Okuma, T.; Lang, S.; Petrov, A.; Weissmüller, J.; Eich, M. Electrochemical Tuning of the Optical Properties of Nanoporous Gold. Sci. Rep. 2017, 7, 44139.

(74) Arnob, M. M. P.; Artur, C.; Misbah, I.; Mubeen, S.; Shih, W. 10X-Enhanced Heterogeneous Nanocatalysis on Nanoporous Gold Disk Array with High-Density Hot Spots. ACS Appl. Mater. Interfaces 2019, 11 (14), 13499-13506.

(75) Kazuma, E.; Jung, J.; Ueba, H.; Trenary, M.; Kim, Y. Direct Pathway to Molecular Photodissociation on Metal Surfaces Using Visible Light. J. Am. Chem. Soc. 2017, 139 (8), 3115-3121.

(76) Kresse, G.; Furthmüller, J. Efficient Iterative Schemes for $a b$ Initio Total-Energy Calculations Using a Plane-Wave Basis Set. Phys. Rev. B: Condens. Matter Mater. Phys. 1996, 54 (16), 11169-11186.

(77) Kresse, G.; Joubert, D. From Ultrasoft Pseudopotentials to the Projector Augmented-Wave Method. Phys. Rev. B: Condens. Matter Mater. Phys. 1999, 59 (3), 1758-1775.

(78) Fang, C.; Li, W.-F.; Koster, R. S.; Klimes, J.; van Blaaderen, A.; van Huis, M. A. The Accurate Calculation of the Band Gap of Liquid Water by Means of GW Corrections Applied to Plane-Wave Density 
Functional Theory Molecular. Phys. Chem. Chem. Phys. 2015, 17, $365-375$.

(79) Foerster, B.; Spata, V. A.; Carter, E. A.; Sönnichsen, C.; Link, S. Plasmon Damping Depends on the Chemical Nature of the Nanoparticle Interface. Sci. Adv. 2019, 5 (3), No. eaav0704.

(80) Olmon, R. L.; Slovick, B.; Johnson, T. W.; Shelton, D.; Oh, S. H.; Boreman, G. D.; Raschke, M. B. Optical Dielectric Function of Gold. Phys. Rev. B: Condens. Matter Mater. Phys. 2012, 86, 235147.

(81) Lai, S. C. S.; Kleyn, S. E. F.; Rosca, V.; Koper, M. T. M. Mechanism of the Dissociation and Electrooxidation of Ethanol and Acetaldehyde on Platinum as Studied by SERS. J. Phys. Chem. C 2008, 112 (48), 19080-19087.

(82) Kresse, G.; Furthmüller, J. Efficiency of ab Initio Total Energy Calculations for Metals and Semiconductors Using a Plane-Wave Basis Set. Comput. Mater. Sci. 1996, 6 (1), 15-50.

(83) Blöchl, P. E. Projector Augmented-Wave Method. Phys. Rev. B: Condens. Matter Mater. Phys. 1994, 50 (24), 17953-17979.

(84) Perdew, J. P.; Burke, K.; Ernzerhof, M. Generalized \{\{Gradient Approximation Made Simple\}\}. Phys. Rev. Lett. 1996, 77 (18), 38653868.

(85) Blöchl, P. E.; Jepsen, O.; Andersen, O. K. Improved Tetrahedron Method for Brillouin-Zone Integrations. Phys. Rev. B: Condens. Matter Mater. Phys. 1994, 49 (23), 16223-16233.

(86) Santiago-Rodrí-Guez, Y.; Herron, J. A.; Curet-Arana, M. C.; Mavrikakis, M. Atomic and Molecular Adsorption on $\{\mathrm{Au}(111)\}$. Surf. Sci. 2014, 627, 57-69.

(87) Shi, H.; Stampfl, C. First-Principles Investigations of the Structure and Stability of Oxygen Adsorption and Surface Oxide Formation at $\{\mathrm{Au}(111)\}$. Phys. Rev. B: Condens. Matter Mater. Phys. 2007, 76 (7), 75327.

(88) Baker, T. A.; Xu, B.; Liu, X.; Kaxiras, E.; Friend, C. M. Nature of Oxidation of the $\mathrm{Au}(111)$ Surface: Experimental and Theoretical Investigation. J. Phys. Chem. C 2009, 113 (38), 16561-16564. 